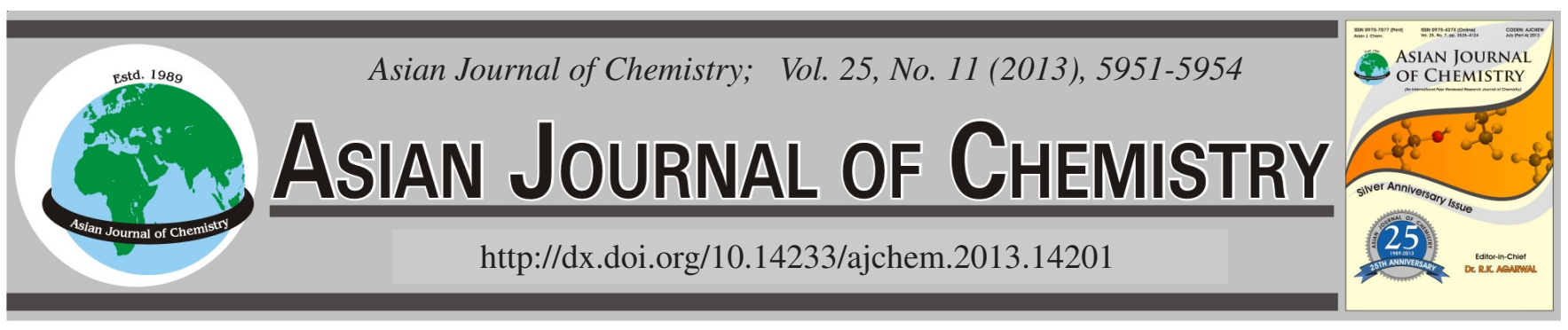

\title{
Extractive Bioconversion of L-Phenylalanine to 2-Phenylethanol Using Polypropylene Glycol 1500
}

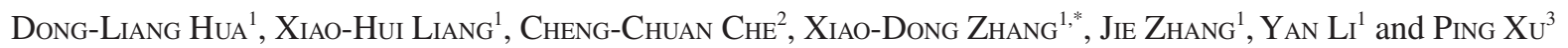

${ }^{1}$ Key Laboratory for Biomass Gasification Technology of Shandong Province, Energy Research Institute of Shandong Academy of Sciences, Jinan 250014, P.R. China

${ }^{2}$ School of Life Sciences, Qufu Normal University, Qufu 273165, P.R. China

${ }^{3}$ School of Life Sciences and Biotechnology, Shanghai Jiao Tong University, Shanghai 200240, P.R. China

*Corresponding author: Fax: +86 531 82961954; Tel: +86 531 85599031; E-mail: hua016@ foxmail.com

\begin{abstract}
Product cytotoxicity was a bottleneck in biotechnological production of 2-phenylethanol from L-phenylalanine. In this study, polypropylene glycol 1500 (PPG 1500) was firstly used as an extractant in the extractive bioconversion of L-phenylalanine by strain Saccharomyces cerevisiae P-3. About $4.8 \mathrm{~g} \mathrm{~L}^{-1}$ 2-phenylethanol was obtained from $7 \mathrm{~g} \mathrm{~L}^{-1} \mathrm{~L}$-phenylalanine in flask experiment within $36 \mathrm{~h}$, with a high molar yield of $92.7 \%$. Compared to conventional bioconversion without addition of extractant, the yield was improved by $26.3 \%$. In a fed-batch extractive bioconversion, the highest concentration of 2-phenylethanol in the organic phase exceeded $22 \mathrm{~g} \mathrm{~L}^{-1}$ and the total concentration in both aqueous and organic phases reached $7.5 \mathrm{~g} \mathrm{~L}^{-1}$. The results showed that the process with polypropylene glycol 1500 as an extractant might have the potential in bioproduction of 2-phenylethanol.
\end{abstract}

Key Words: 2-Phenylethanol, L-phenylalanine, Product cytotoxicity, Polypropylene glycol 1500, Extractive bioconversion.

\section{INTRODUCTION}

In the past decades, whole-cell biocatalysis has emerged as an important tool for the large-scale synthesis of bulk chemicals, agro-chemical intermediates, active pharmaceuticals and food ingredients ${ }^{1}$. According to the FDA and European legislation, products obtained by biotechnological methods can be considered natural, if the substrates for the process are of natural origin $^{2,3}$. Therefore, many studies have been focused on biotechnological production of flavours and fragrances, such as vanillin, $\gamma$-decalactone and 2-phenylethanol (2-PE) $)^{3-6}$.

2-Phenylethanol is an aromatic alcohol with rose-like odours, which occurs in the essential oils of many flowers and plants ${ }^{7}$. Because of its interesting organoleptic characteristic, it is widely used in the cosmetic and perfume industries ${ }^{8}$. Nowadays 2-phenylethanol in the market is mainly produced by chemical synthesis with an annual world production of about 7,000 tons ${ }^{7}$. Natural 2-phenylethanol is extracted from rose petals and due to its rarity, the commercial price is high.

Many types of yeast can synthesize 2-phenylethanol using L-phenylalanine (L-Phe) as substrate through the Ehrlich pathway. Saccharomyces cerevisiae and Kluyveromyces marxianus are known to form important amount of 2-phenylethanol ${ }^{7}$. However, the yield is limited by cytotoxicity of 2-phenylethanol, as well as synergistic inhibitory effects of ethanol ${ }^{9,10}$. To remove the cytotoxicity and enhance 2 -phenylethanol production, in situ product recovery (ISPR) techniques applying different organic solvents as extractants have been reported $^{11,12}$. 2-Phenylethanol is in situ extracted into organic phase, thus decreases the concentration of 2-phenylethanol in aqueous phase and the toxicity to cells. Biocatalytic ability can be maintained from this way and the yield of 2-phenylethanol can be improved. In this study, extractive bioconversion of L-phenylalanine to 2-phenylethanol by strain $S$. cerevisiae P-3 using a novel extractant, polypropylene glycol 1500 (PPG 1500), was investigated.

\section{EXPERIMENTAL}

Oleyl alcohol was purchased from Tokyo Kasei Kogyo Co. Ltd., (Japan). Polypropylene glycol 1500 was product of Jiangsu Hai'an Petrochemical Plant (Jiangsu, China). Lphenylalanine was purchased from Shanghai Kangjie Science of Organism Development Co. Ltd., (Shanghai, China). 2-Phenylethanol, benzyl alcohol and salad oil were obtained from Shanghai Apple Flavour and Fragrance Co. Ltd., (Shanghai, China). Beet molasses from Northeast China included the following compositions (w/w): nonreducing sugars as sucrose $49 \%$, reducing sugars as glucose $0.1 \%$, ashes $8.7 \%$, total nitrogen $2.1 \%$ and available phosphate as $\mathrm{P}_{2} \mathrm{O}_{5} 0.03 \%$. All other chemicals were of analytical grade and were commercially available. 
Strain, medium, culture condition and bioconversion:

The wild strain $S$. cerevisiae P-3 used for all experiments was obtained from our laboratory's collection ${ }^{4}$. This strain was deposited at the China center for type culture collection (CCTCC M 209035).

The yeast cells were first incubated in the seed medium (180 rpm, $35^{\circ} \mathrm{C}$ and $30 \mathrm{~mL}$ in $300-\mathrm{mL}$ flask), which contained $20 \mathrm{gL}^{-1}$ glucose, $2.5 \mathrm{~g} \mathrm{~L}^{-1}$ yeast extract, $2.5 \mathrm{~g} \mathrm{~L}^{-1}$ peptone and $5 \mathrm{~g} \mathrm{~L}^{-1} \mathrm{NaCl}$. The preculture was then inoculated (6\%, v/v) into the bioconversion medium, which was composed of $50 \mathrm{gL}^{-1}$ beet molasses, $7 \mathrm{~g} \mathrm{~L}^{-1} \mathrm{~L}$-phenylalanine, $0.75 \mathrm{~g} \mathrm{~L}^{-1} \mathrm{~K}_{2} \mathrm{HPO}_{4}$ and $0.5 \mathrm{~g} \mathrm{~L}^{-1} \mathrm{KH}_{2} \mathrm{PO}_{4}$.

Extractive bioconversion was carried out with the addition of different organic solvents as extractants. These extractants were added directly with an aqueous/organic ration of 2:1 (v/v) when the bioconversion started. Controls were conducted without addition of any solvent. The bioconversion was carried out at $180 \mathrm{rpm}, 35^{\circ} \mathrm{C}$ and $50 \mathrm{~mL}$ in 500-mL flask. At the end of the bioconversion, samples were taken and concentrations of 2-phenylethanol in both organic and aqueous phases were measured.

Fed-batch extractive bioconversion was carried out in a 30-L bioreactor (equipped with Biotech-2002 bioprocess controller, Shanghai Baoxing Bio-engineering Equipment Co. Ltd., China). The $\mathrm{pH}$ was controlled between $5.0-5.8$ by automatically adding $10 \mathrm{~mol} \mathrm{~L}^{-1} \mathrm{NaOH}$ with a peristaltic pump. Vigorous agitation (stirring at $600 \mathrm{rpm}$ ) and aeration (air flow at $2.0 \mathrm{vvm}$ ) were maintained to keep a good oxygen supply. Additional L-phenylalanine and beet molasses were added to the fermentation broth without sterilization during the bioconversion. At regular time intervals, samples were taken to determine the concentrations of 2-phenylethanol, L-phenylalanine and $\mathrm{pH}$.

Analytical methods: The $\mathrm{pH}$ of the culture was determined by Orion $828 \mathrm{pH}$ meter (Orion Research, Inc.).

Concentrations of 2-phenylethanol were analyzed by a VARIAN-3380 gas chromatography (GC), equipped with a SPB-5 capillary column $(30 \mathrm{~m} \times 0.25 \mathrm{~mm} \times 0.25 \mu \mathrm{m}$, Supelco, USA). Before GC analysis samples were centrifuged at 12,000 rpm for $10 \mathrm{~min}$. The organic phase was diluted with butyl acetate $(1: 10, \mathrm{v} / \mathrm{v})$ with the addition of benzyl alcohol as internal standard. The temperatures of the injector and flame ionization detector were both $280^{\circ} \mathrm{C}$. The column oven temperature was maintained at $160^{\circ} \mathrm{C}$ for $8 \mathrm{~min}$. The retention times of benzyl alcohol and 2-phenylethanol were $3.3 \mathrm{~min}$ and 3.6 min, respectively. Quantitative data were obtained by comparing the peak areas of the query compounds with those of standards of known concentrations.

Concentrations of L-phenylalanine were estimated by high-performance liquid chromatography (HPLC, Agilent 1100 series, Hewlett-Packard). Before HPLC analysis samples were centrifuged at $12,000 \mathrm{rpm}$ for $10 \mathrm{~min}$. The aqueous phase was diluted with distilled water and filtered with aqueous phase filter film of $0.45 \mu \mathrm{m}$. The HPLC system was equipped with a KR100-5 $\mathrm{C}_{18}$ column $(150 \mathrm{~mm} \times 4.6 \mathrm{~mm} \times 5 \mu \mathrm{m}$, Kromasil, Sweden) and a UV detector operating at a wavelength of 260 $\mathrm{nm}$. The column was eluted with a mixture of methanol and water $(60: 40, \mathrm{v} / \mathrm{v})$ at a flow rate of $0.5 \mathrm{~mL} \mathrm{~min}^{-1}$. The retention time of L-phenylalanine was 3.33 min. Quantitative data were obtained by comparing the peak areas of the query compounds with those of standards of known concentrations.

\section{RESULTS AND DISCUSSION}

Selection of extractants: Among the criteria for the selection of suitable extractants, distribution coefficient and biocompatibility are considered to be crucial. In extractive bioconversion, the selected organic solvent needs to have a favourable distribution coefficient for target product and continues to extract the product into organic phase. The toxicity of organic solvent to yeast cells should also be taken into account. Partition coefficient is the ratio of concentrations of a compound in the two phases of a mixture of two immiscible solvents at equilibrium, in which these two solvents are normally water and octanol. The value of the logarithm of partition coefficient $(\log \mathrm{P})$, was used to select the potential extractant in this study ${ }^{13}$. It is considered that organic solvents with $\log \mathrm{P}$ values lower than 5 such as ethyl acetate $(\log \mathrm{P}=$ $0.7)$, hexanol $(\log \mathrm{P}=1.8)$ and octane $(\log \mathrm{P}=4.5)$, show high toxicity to yeast cells. Therefore, we only chose organic solvents with $\log \mathrm{P}$ values higher than 5 other than those reported. On the other hand, these extractants should be stable, inexpensive and available in bulk quantities.

Extractive bioconversion using oleic acid $(\log \mathrm{P}=7.7)$, oleyl alcohol $(\log \mathrm{P}=7.5)$, isopropyl myristate $(\log \mathrm{P}=7.4)$, salad oil and polypropylene glycol 1500 as extractants was carried out. After a 36-h cultivation, concentrations of 2-phenylethanol in both aqueous and organic phases were measured, respectively. Fig. 1 showed total 2-phenylethanol production from the extractive bioconversion using different solvents. All of the extractive bioconversion obtained higher 2-phenylethanol yield than the controls. Polypropylene glycol 1500 was the best, from which about $4.8 \mathrm{~g} \mathrm{~L}^{-1}$ 2-phenylethanol was achieved with a high molar yield of $92.7 \%$. Contrasted to the controls without addition of extractant $\left(3.8 \mathrm{~g} \mathrm{~L}^{-1} 2\right.$ phenylethanol obtained), the yield was improved by $26.3 \%$. The widely reported oleic acid was the worst, from which only $4.12 \mathrm{~g} \mathrm{~L}^{-1}$ 2-phenylethanol was obtained. Based on the above results, it is concluded that polypropylene glycol 1500 was the most suitable.

Fed-batch extractive bioconversion: Fed-batch extractive bioconversion with polypropylene glycol 1500 as extractant was then performed. Polypropylene glycol 1500 (4 L) was added to the bioconversion medium $(14 \mathrm{~L})$ when the fermentation started (aqueous/organic ratio $=3.5: 1, \mathrm{v} / \mathrm{v}$ ). As Fig. 2 showed, L-phenylalanine was gradually consumed while the concentration of 2-phenylethanol increased. The highest concentration of 2-phenylethanol was obtained at $38 \mathrm{~h}$. The concentrations of 2-phenylethanol in the aqueous and organic phase were $1.14 \mathrm{~g} \mathrm{~L}^{-1}$ and $22 \mathrm{~g} \mathrm{~L}^{-1}$, respectively. Calculated on the initial aqueous medium, the total 2-phenylethanol produced was $7.5 \mathrm{~g} \mathrm{~L}^{-1}$, with a molar yield of $78 \%$. The increase of 2-phenylethanol (including in the organic phase and in the aqueous phase) was growth-associated, which was similar to conventional bioconversion (data not shown). During the process the concentrations of 2-phenylethanol in the aqueous phase remained below $1.3 \mathrm{~g} \mathrm{~L}^{-1}$. 


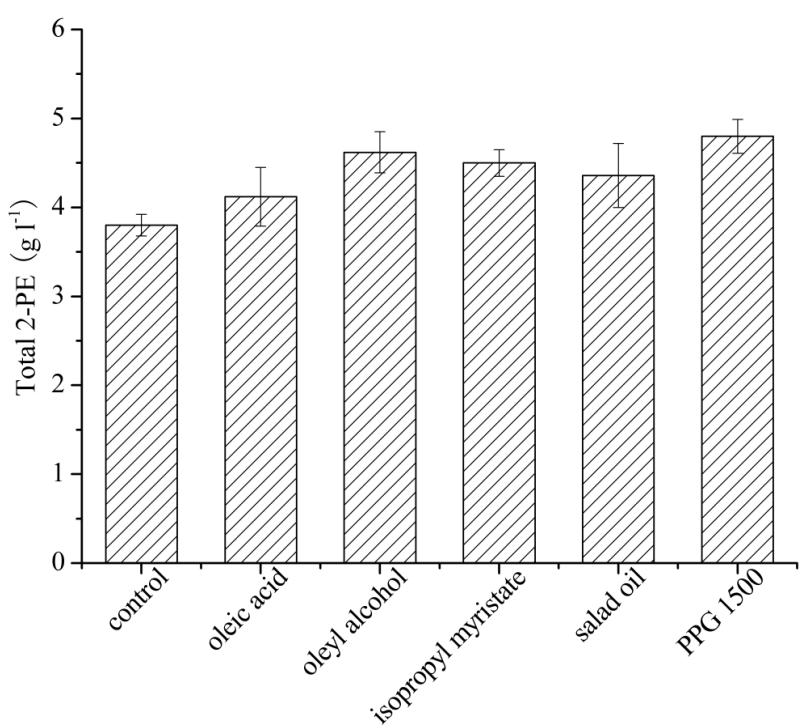

Fig. 1. Total concentration of 2-phenylethanol obtained in the extractive bioconversion using different extractants. These extractants were directly added at an aqueous/organic ratio of $2: 1$. The bioconversion was carried out on a rotary shaker with $180 \mathrm{rpm}$ at $35^{\circ} \mathrm{C}$ and $50 \mathrm{~mL}$ medium in 500-mL flask. The values were means of three replicates and the error bars indicated standard deviations

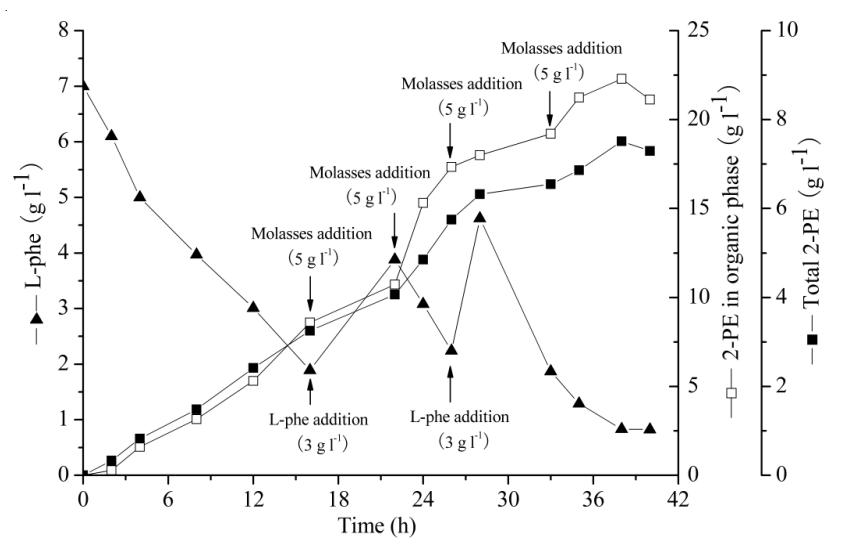

Fig. 2. Fed-batch extractive bioconversion of L-phenylalanine to 2 phenylethanol using polypropylene glycol 1500 in a 30-L bioreactor. Polypropylene glycol 1500 (4 L) was added to the aqueous medium $(14 \mathrm{~L})$ when the biocoversion started (aqueous/organic ratio $=3.5: 1$, $\mathrm{v} / \mathrm{v}$ ). The $\mathrm{pH}$ was controlled between $5.0-5.8$ by automatically adding $10 \mathrm{~mol} \mathrm{~L}^{-1} \mathrm{NaOH}$ with a peristaltic pump. Vigorous agitation (stirring at $600 \mathrm{rpm}$ ) and aeration (air flow at $2.0 \mathrm{vvm}$ ) were maintained to keep a good oxygen supply. Additional L-phenylalanine and beet molasses were added to the fermentation broth without sterilization. Symbols: $\boldsymbol{\Delta}$ L-phenylalanine, $\square$ 2-phenylethanol in organic phase, $\square$ total 2-phenylethanol

Solvent selection strategies for extractive biocatalysis and desirable solvent characteristics had been reviewed ${ }^{13,14}$. The choice of suitable solvent was based on following criteria: favourable distribution coefficient for product, high selectivity, low emulsion-forming tendency, low aqueous solubility, chemical and thermal stability, favourable properties for product recovery, nonbiodegradability, nonhazardous, inexpensive, available in bulk quantities and biocompatibility. Oleic acid, oleyl alcohol, isopropylmyristate, miglyol and polypropylene glycol 1200 have been reported used in the extractive bioconversion of L-phenylalanine for 2-phenylethanol production.
Among them, oleic acid was the most widely applied ${ }^{7,11,12,15}$. The concentration of 2-phenylethanol reached $2.21 \mathrm{~g} \mathrm{~L}^{-1}$ by strain $S$. cerevisiae HJ using oleic acid as extractant, which was improved by $8.6 \%$ contrasted to single phase bioconver$\operatorname{sion}^{16}$. The same bioconversion process was carried out by strain S. cerevisiae CICIM Y0086(T) and $3 \mathrm{~g} \mathrm{~L}^{-1}$ 2-phenylethanol was obtained when the volume ration of organic phase to aqueous phase was $1: 1^{17}$. Compared to monophasic aqueous system the concentration of 2-phenylethanol was improved by $50 \%$. In the extractive bioconversion by strain $S$. cerevisiae Giv 2009, the overall 2-phenylethanol concentration in the oleic phase reached $12.6 \mathrm{~g} \mathrm{~L}^{-1}$ as the 2-phenylethanol concentration in the oleic phase was $24 \mathrm{~g} \mathrm{~L}^{-115}$. An average spacetime yield, $0.26 \mathrm{~g} \mathrm{~L}^{-1} \mathrm{~h}^{-1}$, was achieved. Though 2-phenylethanol production using oleic acid as extractant was higher than that of polypropylene glycol in this study, the main drawback of the process applying oleic acid lies in that the addition of oleic acid may make troubles in the product recovery process. For example, the boiling point of oleic acid is $286^{\circ} \mathrm{C}(100 \mathrm{~mm} \mathrm{Hg})$ and the boil point of 2-phenylethanol is $220^{\circ} \mathrm{C}$. Though 2-phenylethanol can be separated from oleic acid by vacuum distillation, the alcohol (2-phenylethanol) and the acid (oleic acid) may react at high temperature, which made oleic acid unsuitable for industrial application.

Unlike oleic acid, polypropylene glycol 1500 has a good chemical and thermal stability, which will not evaporate, decompose, or react with the target product at high temperature during vacuum distillation. In this study, polypropylene glycol 1500 was firstly used as an extractant in the extractive bioconversion of L-phenylalanine to decrease the cytotoxicity of target product and enhance the yield. Higher 2-phenylethanol production and molar yield were obtained by this way. The total 2-phenylethanol produced was $7.5 \mathrm{~g} \mathrm{~L}^{-1}$, with a molar yield of $78 \%$. Etschmann and Schrader ${ }^{11}$ reported a similar 2-phenylethanol production process using polypropylene glycol 1200 as in situ extractant. L-phenylalanine was used as the sole nitrogen source and saturated in the medium. With $K$. marxianus CBS 600, in a fed-batch bioconversion $26.5 \mathrm{~g} \mathrm{~L}^{-1}$ 2-phenylethanol was produced in the organic phase, corresponding to the space-time yield of $0.33 \mathrm{~g} \mathrm{~L}^{-1} \mathrm{~h}^{-1}$. The total 2-phenylethanol concentration reached $10.2 \mathrm{~g} \mathrm{~L}^{-1}$, with a more yield of about $25 \%$. 2-Phenylethylacetate was found to be the main byproduct, the total concentration of which was $2.3 \mathrm{~g} \mathrm{~L}^{-1}$. Though the space-time yield in this study was lower $(0.197 \mathrm{~g}$ $\mathrm{L}^{-1} \mathrm{~h}^{-1}$ ) than the process by strain $K$. marxianus CBS 600, the molar yield (78\%) was much higher. On the other way, contrasted to polypropylene glycol 1200 used in the extractive bioconversion by the strain K. marxianus CBS 600, polypropylene glycol 1500 has a larger molecular weight, which may have a lower water solubility and emulsion-forming tendency ${ }^{11}$. Separation of the aqueous and organic phases thus becomes easier. As an inexpensive commodity which is available in bulk quantities, the result shown in this study will make polypropylene glycol 1500 suitable for the large scale application in the food industry for 2-phenylethanol production by bioconversion. Therefore, the process with polypropylene glycol 1500 as an extractant might have a better potential in bioproduction of 2-phenylethanol. 


\section{ACKNOWLEDGEMENTS}

The work was supported by the Young Scientists' Program of National Natural Science Foundation of China (Grant 31100030), the Excellent Middle-Aged and Youth Scientist Award Foundation of Shandong Province (Grant BS2011SW033), the Foundation for Development of Science and Technology of Shandong Academy of Sciences (Grant 201114) and State Key Laboratory of Microbial Technology of Shandong University (M2011-17). Mr. Che also thanks the supports by the Qufu Normal University Foundation (XJ201003).

\section{REFERENCES}

1. P. Xu, J.H. Qiu, Y.N. Zhang, J. Chen, P.G. Wang, B. Yan, J. Song, R.M. Xi, Z.X. Deng and C.Q. Ma, Adv. Synth. Catal., 349, 1614 (2007)

2. D.L. Hua, C.Q. Ma, S. Lin, L.F. Song, Z.X. Deng, Z. Maomy, Z.B. Zhang, B. Yu and P. Xu, J. Biotechnol., 130, 463 (2007).

3. P. Xu, D.L. Hua and C.Q. Ma, Trends Biotechnol., 25, 571 (2007).

4. D.L. Hua, S. Lin, Y.F. Li, Z.B. Zhang, Y. Du, H. Chen, X.H. Zhang and P. Xu, Biocatal. Biotransfor., 28, 259 (2010).
5. D.L. Hua, C.Q. Ma, L.F. Song, S. Lin, Z.B. Zhang, Z.X. Deng and P. Xu, Appl. Microbiol. Biotechnol., 74, 78 (2007).

6. J. Schrader, M.M.W. Etschmann, D. Sell, J.M. Hilmer and J. Rabenhorst, Biotechnol. Lett., 26, 463 (2004).

7. M.M.W. Etschmann, W. Bluemke, D. Sell and J. Schrader, Appl. Microbiol. Biotechnol., 59, 1 (2002).

8. C.J. Huang, S.L. Lee and C.C. Chou, Food Res. Int., 34, 277 (2001).

9. G. Lester, J. Bacteriol., 90, 29 (1965).

10. D. Stark, D. Zala, T. Münch, B. Sonnleitner, I.W. Marison and U. von Stockar, Enzym. Microb. Technol., 32, 212 (2003).

11. M.M.W. Etschmann and J. Schrader, Appl. Microbiol. Biotechnol., 71, 440 (2006).

12. D. Serp, U. von Stockar and I.W. Marison, Biotechnol. Bioeng., 82, 103 (2003).

13. L.J. Bruce and A.J. Daugulis, Biotechnol. Progr., 7, 116 (1991).

14. R. León, P. Fernandes, H.M. Pinheiro and J.M.S. Cabral, Enzym. Microb. Technol., 23, 483 (1998).

15. D. Stark, T. Münch, B. Sonnleitner, I.W. Marison and U. von Stockar, Biotechnol. Progr., 18, 514 (2002).

16. C.T. Wang, B.G. Sun, Y.P. Cao, J. Wang and H. Zhang, Modern Chem. Ind., 28, 38 (2008)

17. J. Lu and W.G. Zhang, Chem. Ind. Eng. Progr., 27, 417 (2008). 